\title{
Der Sonderbeitrag «HIN-Abonnement für alle» läuft ab. Was bedeutet das?
}

Jacques de Haller, Präsident FMH - Annamaria Müller Imboden, Generalsekretärin FMH

Die Ärztekammer hat im vergangenen Jahr beschlossen, den Sonderbeitrag von Fr. 50.- für das «HIN-Abonnement für alle» in diesem Jahr zum letzten Mal zu erheben. Mit Hilfe dieses Sonderbeitrags konnte während fünf Jahren allen FMHMitgliedern ein HIN-Basisabonnement kostenlos zur Verfügung gestellt werden. Der Wegfall dieser Pauschalfinanzierung bedeutet nun, dass ab dem kommenden Jahr jede Ärztin und jeder Arzt für die Kosten ihres oder seines HIN-Abonnements direkt aufzukommen hat.

Die FMH hat 1996 das Ziel definiert, die Nutzung des Internets in Arztpraxen zu fördern. $\mathrm{Zu}$ diesem Zweck wurde die Firma Health Info Net AG (HIN) gegründet. HIN hatte die Aufgabe, die Infrastuktur und die Technologien bereitzustellen, um eine rechtskonform gesicherte Datenübertragung im Gesundheitsbereich zu ermöglichen. Eine Aufgabe, der HIN sich in den darauffolgenden Jahren mit grossem Einsatz erfolgreich stellte. Mit der Sonderbeitragsaktion, welche 2001 begann, konnte ein grosser Teil der Ärztinnen und Ärzte von den vielfältigen Vorteilen der Nutzung der HIN-Plattform überzeugt werden. Auch und nicht zuletzt diese Tatsache gab einen wesentlichen Impuls zur Erreichung des ursprünglich gesetzten Zieles.
Mit rund 10000 angeschlossenen Ärztinnen und Ärzten, über 30 Spitälern sowie verschiedenen Spital- und Gesundheitsnetzen ist HIN heute zu einer anerkannten E-Health-Plattform im Schweizer Gesundheitswesen geworden, die sich über eine vielfältige Palette von Dienstleistungen selbst finanziert. Die Fortsetzung der Unterstützung durch eine Pauschalfinanzierung ist deshalb weder notwendig noch sinnvoll. Ab 2006 werden die FMH-Mitglieder die von ihnen bezogenen Dienstleistungen mittels Rechnung an das HIN bezahlen und nicht mehr, wie bis anhin, im Rahmen ihrer Mitgliederbeiträge an die FMH. HIN wird seine Kunden hierüber noch detailliert informieren.

Die FMH hat sich mit der Gründung von HIN visionäre Ziele gesetzt. Mit Freude nehmen wir zur Kenntnis, dass diese Ziele erreicht werden konnten. Wir sind überzeugt, dass sich für die FMH mit der HIN-Plattform noch weitere Synergien erschliessen lassen. In den nun folgenden Monaten werden die Fragen um künftige, zukunftsgerichtete Formen der Zusammenarbeit intensiv bearbeitet und anschliessend der Ärztekammer zur Beschlussfassung vorgelegt. 